\title{
Drastic shrinking of the Hadley circulation during the mid-Cretaceous Supergreenhouse
}

\author{
H. Hasegawa, R. Tada, X. Jiang, Y. Suganuma, S. Imsamut, C. Punya, N. Ichinnorov, Y. Khand
}

\section{Supplementary Material}

Table S1: Paleontological age constraints of the Cretaceous terrestrial deposits in the Asian interior basins

Table S2: Stratigraphic compilation of the climate-sensitive sediments with special emphasis on desert (eolian dune) deposits cited in Figure 3.

Supplementary Methods: Paleoposition and Age constraints for the eolian sandstone formations in the Asian interior basins

Figure S1: Lithostratigraphic column, paleowind direction data, and magnetic polarity sequence of the eolian sandstone deposits (Jiaguan Formation) in Sichuan Basin, south China

Figure S2: Lithostratigraphic column, paleowind direction data, and magnetic polarity sequence of the eolian sandstone deposits (Phu Thok Formation) in the Khorat Basin, northeast Thailand

\section{Supplementary References}


Table S1: Paleontological age constraints of the Cretaceous terrestrial deposits in the Asian interior basins based on the fossil assemblages of ostracods, charophytes, pollen and spores (modified after, Li, 1982; Racey et al., 1996; Hao et al., 2000; Khand et al., 2000; Meesok, 2000; Chen et al., 2006; Sha, 2007; Hasegawa et al., 2010).

\section{Gobi Basin}

\begin{tabular}{|c|c|c|c|}
\hline Formation & Lithology & Fossil assemblage & Age \\
\hline Tsagantsav Fm & $\begin{array}{l}\text { Reddish brown to whitish grey, } \\
\text { conglomerate and sandstone, } \\
\text { reddish brown mudstone, } \\
\text { calcretes, basalts }\end{array}$ & $\begin{array}{l}\text { Ostracodes: Cypridea unicostata } \\
\text { Plants: Baiera manchurica, Cladophiledis onychiopsis, } \\
\text { Nilssoniopteris denticulata }\end{array}$ & Ber.-Brm. \\
\hline Shinehudag Fm & $\begin{array}{l}\text { Greenish grey to whitish grey, } \\
\text { siltstone and mudstone }\end{array}$ & $\begin{array}{l}\text { Ostracodes: Cypridea fasciculata } \\
\text { Charophytes: Aclistochara caii, Raskyella sp. } \\
\text { Pollen and Spores: Cicatricosisporites australiensis, } \\
\text { Densoisporites velatus, Pilosisporites trichopapilosus }\end{array}$ & Brm?-Apt. \\
\hline Khukhteeg Fm & $\begin{array}{l}\text { Whitish grey to brown, } \\
\text { conglomerate, sandstone, } \\
\text { mudstone, and lignites }\end{array}$ & $\begin{array}{l}\text { Ostracodes: Cypridea acutituberculata, Tsetsenia } \\
\text { mira, Trapeoidella khandae, Janinella tsaganensis } \\
\text { Charophytes: Atopochara trivolvis, Mesochara voluta, } \\
\text { M. tuzsoni } \\
\text { Pollen and Spores: Foraminisporites assemmetricus, } \\
\text { Alisporites elongatus, Abiespollenites sp. }\end{array}$ & Alb. \\
\hline $\begin{array}{l}\text { Bayanshiree } \\
\text { Fm }\end{array}$ & $\begin{array}{l}\text { Reddish brown to whitish grey, } \\
\text { conglomerate, sandstone, } \\
\text { mudstone, and calcretes }\end{array}$ & $\begin{array}{l}\text { Ostracodes: Lycopterocypris baishintsavica, } \\
\text { Charophytes: Atopochara multivolvis, Caucasuella } \\
\text { gulistanica }\end{array}$ & Cen.-San. \\
\hline Djadokhta Fm & $\begin{array}{l}\text { Reddish to reddish brown, } \\
\text { eolian sandstone and mudstone }\end{array}$ & Ostracodes: Gobiocypris tugrigensis & e. Camp. \\
\hline Barungoyot Fm & $\begin{array}{l}\text { Reddish to reddish brown, } \\
\text { eolian sandstone and mudstone }\end{array}$ & Ostracodes: Talicypridea abdarantica & l. Camp. \\
\hline Nemegt Fm & $\begin{array}{l}\text { Reddish brown to whitish grey, } \\
\text { conglomerate, sandstone, } \\
\text { mudstone, and calcretes }\end{array}$ & $\begin{array}{l}\text { Ostracodes: Talicypridea reticulata, Mongolocypris } \\
\text { distributa } \\
\text { Charophytes: Mongololiachara mesochara }\end{array}$ & e. Maas. \\
\hline Dzunmod Fm & $\begin{array}{l}\text { Reddish brown to whitish grey, } \\
\text { eolian sandstone, mudstone, and } \\
\text { calcretes }\end{array}$ & no fossils & m. Maas. \\
\hline
\end{tabular}

\section{Ordos Basin}

\begin{tabular}{|c|c|c|c|}
\hline Formation & Lithology & Fossil assemblage & Age \\
\hline Luohe Fm & $\begin{array}{l}\text { Red to purple, } \\
\text { medine- to } \\
\text { sandstone }\end{array}$ & $\begin{array}{l}\text { Ostracodes: Darwinula contracta } \\
\text { Vertebrates: Lycoptera sp. }\end{array}$ & Ber.-Vlg. \\
\hline $\begin{array}{l}\text { Huanhe Fm } \\
\text { (Huachi Fm) }\end{array}$ & $\begin{array}{l}\text { Yellowish-green } r \\
\text { reddish-purple, fine-grained } \\
\text { sandstone, siltstone, and } \\
\text { mudstone }\end{array}$ & $\begin{array}{l}\text { Ostracodes: Cypridea unicostata, C. koskule- } \\
\text { nsis, Rhinocypris cirrita } \\
\text { Dinosaurs: Psittacosaurus sp. }\end{array}$ & Vlg. \\
\hline $\begin{array}{l}\text { Luohangdong } \\
\text { Fm }\end{array}$ & $\begin{array}{l}\begin{array}{l}\text { Red to purple, } \\
\text { mediume- to } \\
\text { sandstone }\end{array} \\
\text { sained, }\end{array}$ & $\begin{array}{l}\text { Ostracodes: Cypridea vitimensis, C. consulta, C. } \\
\text { koskulensis, Clinocypris scolia, Rhinocypris cirrita, } \\
\text { Lycopterocypris infantilis, Darwinula simplus, } \\
\text { Rhinocypris foveata, Djungarica stolida } \\
\text { Dinosaurs: Psittacosaurus youngi }\end{array}$ & Vlg.-Hau. \\
\hline Jingchuang Fm & $\begin{array}{l}\text { Yellowish-green } r \text { to } \\
\text { reddish-purple, fine-grained } \\
\text { sandstone, siltstone, and } \\
\text { mudstone }\end{array}$ & $\begin{array}{l}\text { Ostracodes: Cypridea unicostata, C. yumenensis, C. } \\
\text { justa, C. koskulensis C. consulta, C. subrostrata, } \\
\text { Pseudocypridina globra, Clinocypris scolia, } \\
\text { Lycopterocypris infantilis, Jungarica stolida, Rhinocypris }\end{array}$ & Hau.-Brm. \\
\hline
\end{tabular}




\begin{tabular}{|l|l|l|l|}
\hline & & $\begin{array}{l}\text { foveata, R. cirrita } \\
\text { Pollen and Spores: Cicatricosis porites, Densois } \\
\text { porites, Piceae pollenites } \\
\text { Dinosaurs: Psittacosaurus youngi }\end{array}$ & \\
\hline Lamawan Fm & $\begin{array}{l}\text { Yellowish-grey to } \\
\text { whitish-grey, medium- to } \\
\text { coarce-grained sandstone, } \\
\text { mudstone, and coals }\end{array}$ & $\begin{array}{l}\text { Plants: Elatocladus manchuricus, E. obtusifolia, } \\
\text { Brachyphyllum japonicum, Sphenolepidium sp., } \\
\text { Coniopteris onychioides, Czekanowskia rigida, } \\
\text { Podozamites lanoeolatus, Stenorachis bulunensis }\end{array}$ & \\
\hline Tegaimiao Fm & $\begin{array}{l}\text { Red to orange, fine- to } \\
\text { medium-grained, eolian } \\
\text { sandstone }\end{array}$ & Dinosaurs: Protoceratops sp. & San.-Camp. \\
\end{tabular}

\section{Tarim Basin}

\begin{tabular}{|c|c|c|c|}
\hline Formation & Lithology & Fossil assemblage & Age \\
\hline Kezilesu Gp & $\begin{array}{l}\text { Red to purple, conglomerate, } \\
\text { sandstone, and sandy } \\
\text { mudstone }\end{array}$ & 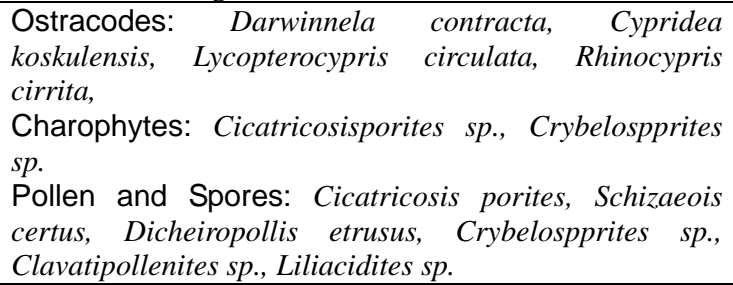 & Ber.-Brm. \\
\hline Kumutake Fm & $\begin{array}{l}\text { Red to } \begin{array}{l}\text { orange, pebbly } \\
\text { sandstone, sandstone, and } \\
\text { mudstone }\end{array} \\
\end{array}$ & $\begin{array}{l}\text { Ostracodes: Talicypridea meliora, T. gemma, Cypridea } \\
\text { cavernosa, C. rostrata, Ziziphocypris simakovi, } \\
\text { Candoniella mordvilkoi }\end{array}$ & Con.-Camp. \\
\hline Subashi Fm & $\begin{array}{l}\text { Whitish-grey to reddish, } \\
\text { medium to coarse-grained } \\
\text { sandstone, and mudstone }\end{array}$ & $\begin{array}{l}\text { Ostracodes: Cypridea mosuowanensi, Talicypridea } \\
\text { amoena, T. gemma, T. retusa } \\
\text { Dinosaurs: Tarbosaurus sp., Nemegtosaurus pachi, } \\
\text { Oolithes spherides, Mongolimys turfanensis, } \\
\text { Shanshanosaurus huoyanshanensis }\end{array}$ & Camp.-Maas. \\
\hline
\end{tabular}

\section{Subei Basin}

\begin{tabular}{|c|c|c|c|}
\hline Formation & Lithology & Fossil assemblage & Age \\
\hline Xihengshan Fm & $\begin{array}{l}\text { Yellowish grey to purpe, } \\
\text { sandstone, mudstone, and } \\
\text { coals }\end{array}$ & $\begin{array}{l}\text { Ostracodes: Cypridea sp. } \\
\text { Plants: Gleichenites nipponensis, Brachyphyllum besum, } \\
\text { Sphenopteris nitidula }\end{array}$ & Ber.-Vlg. \\
\hline $\begin{array}{l}\text { Longwangshan } \\
\text { Fm }\end{array}$ & $\begin{array}{l}\text { Whitish grey to purple, } \\
\text { andesite lava, and andesitic } \\
\text { tuff breccia }\end{array}$ & no fossils & Vlg. \\
\hline $\begin{array}{l}\text { Dawangshan } \\
\text { Fm }\end{array}$ & $\begin{array}{l}\text { Purple to grey, andesitic tuff } \\
\text { breccia }\end{array}$ & $\begin{array}{l}\text { Plants: Pagiophyllum sp., Potozamites sp., Otozamites } \\
\text { sp. }\end{array}$ & Hau.-Brm. \\
\hline Gecun Fm & $\begin{array}{llr}\text { Reddish } & \text { orange, } & \text { greenish } \\
\text { grey, } & \text { sandstone, } & \text { and } \\
\text { mudstone } & & \end{array}$ & $\begin{array}{l}\text { Charophytes: Sphaerochara vertieillata, S. stontoni, } \\
\text { Charites symmetrica, Flabellochara jurongica } \\
\text { Pollen and Spores: Classopollis sp., Cicatricosisporites } \\
\text { sp. } \\
\text { Plants: Frenelopsis sp. }\end{array}$ & Apt.-Alb. \\
\hline Pukou Fm & $\begin{array}{l}\text { Reddish-purple to grey, } \\
\text { pebbly sandstone }\end{array}$ & $\begin{array}{l}\text { Ostracodes: Cypridea sp., Talicypridea sp., Ziphocypris } \\
\text { simakovi } \\
\text { Pollen and Spores: Hizaeois porites, Welwitshia pites }\end{array}$ & Cen.-Con. \\
\hline Chishan Fm & $\begin{array}{l}\text { Reddish-purple } \\
\text { greenish-grey, sandstone and } \\
\text { mudstone }\end{array}$ & $\begin{array}{l}\text { Ostracodes: Cypridea cavernosa, Talicypridea sp., } \\
\text { Eucypris sp. } \\
\text { Plants: Manica tholistoma }\end{array}$ & San.-Maas. \\
\hline
\end{tabular}

\section{Jianghan Basin}

\begin{tabular}{|l|l|l|l|}
\hline Formation & Lithology & Fossil assemblage & Age \\
\hline Wulong Fm & Reddish to yellowish-grey, & Ostracodes: Cypridea prognata, Mantelliana gigantea, & Alb. \\
\hline
\end{tabular}




\begin{tabular}{|c|c|c|c|}
\hline & $\begin{array}{l}\text { conglomerate, sandstone, } \\
\text { mudstone }\end{array}$ & 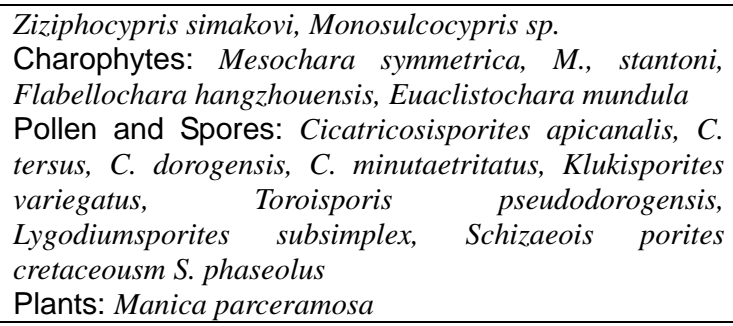 & \\
\hline Luojinghu Fm & $\begin{array}{l}\text { Reddish-purple to grey, } \\
\text { conglomerate, sandstone }\end{array}$ & Ostracodes: Cypridea cavernosa & Cen.-Tur. \\
\hline Honghuatao Fm & $\begin{array}{l}\text { Reddish to yellowish orange, } \\
\text { fine-grained eolian sandstone }\end{array}$ & $\begin{array}{l}\text { Ostracodes: Cypridea cavernosa, Talicypridea amoena, } \\
\text { T. longa } \\
\text { Charophytes: Porochara anluensis }\end{array}$ & Con.-San. \\
\hline Paomagang Fm & $\begin{array}{l}\text { Reddish to whitish grey, } \\
\text { sandstone and mudstone }\end{array}$ & $\begin{array}{l}\text { Ostracodes: Cypridea cavernosa, C. nanxiongensis, C. } \\
\text { tera, Talicypridea amoena, T. longa, T. chinensis, T. } \\
\text { quadrata } \\
\text { Charophytes: Latochara cylindrica, L. curtula, } \\
\text { L. yunnanensis, Peckichara dangyangensis, } \\
\text { Charites tenuis }\end{array}$ & Camp.-Maas. \\
\hline
\end{tabular}

\section{Sichuan Basin}

\begin{tabular}{|c|c|c|c|}
\hline Formation & Lithology & Fossil assemblage & Age \\
\hline Tianmashan Fm & $\begin{array}{l}\text { Reddish-purple conglomerate, } \\
\text { sandstone, and mudstone }\end{array}$ & $\begin{array}{l}\text { Ostracodes: Deyangia lushanensis, D. postacuta, } \\
\text { Cypridea sp., Jingguella obtusura, Lycopterocypris sp., } \\
\text { Minheella sp., Ziziphocypris sp., Mongolianella sp. }\end{array}$ & Ber.-Brm. \\
\hline Jiaguan Fm & $\begin{array}{lr}\text { Reddish-purple, } & \text { fine- } r \\
\text { medium-grained, } & \text { eolian } \\
\text { sandstone } & \end{array}$ & $\begin{array}{l}\text { Ostracodes: Cypridea angusticaudata, C. sichuanensis, } \\
\text { C. yunnanensis, C. gunzulingensis, C. enodata, C. cf. } \\
\text { ampullaceousa, C. concise, C. tera, C. cf. gibbosa, C. } \\
\text { setina frorida, C. sentina acerata, C. setina bellatula, C. } \\
\text { (Bisulcocypridea) sp., C. (B.) chuxiongensis, C. } \\
\text { (Morinina) monosulcata, Harbinia jingshanensin, Latonia } \\
\text { (Monosulcocypris) spp., Sinocypris (Quadracypris) cf. } \\
\text { favosa, Talicypridea (Cristocypridea) sp., Ziziphocypris } \\
\text { orbita, Z. acuta, Jinggunella sp., Kaitunia } \\
\text { cuneata,Darwinnela sp., Timiriasevia sp., Lycopterocypris } \\
\text { sp., Pinnocypridea sp. }\end{array}$ & Apt.-Tur. \\
\hline Guankou Fm & $\begin{array}{l}\text { Red to purple, fine-grained } \\
\text { sandstone, mudstone, gypsum }\end{array}$ & 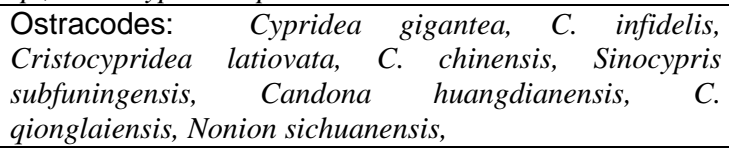 & Con.-Maas. \\
\hline
\end{tabular}

\section{Simao Basin}

\begin{tabular}{|c|c|c|c|}
\hline Formation & Lithology & Fossil assemblage & Age \\
\hline Jingxing Fm & $\begin{array}{l}\text { Yellowish-grey to } \\
\text { whitish-grey, sandstone, } \\
\text { mudstone, and coals }\end{array}$ & $\begin{array}{l}\text { Ostracodes: Monosulcocypris reticulata, Cyp- } \\
\text { ridea angusticaudata, Candona yunnanensis, } \\
\text { Rhinocypris tuberculata, Limnocythere tumulosa }\end{array}$ & Ber.-Brm. \\
\hline Nanxin Fm & $\begin{array}{l}\text { Red to purple, sandstone, and } \\
\text { mudstone }\end{array}$ & $\begin{array}{l}\text { Ostracodes: Monosulcocypris subovata, M. subelliptica, } \\
\text { M. longa, M. gigantea, M. yunnanensis, M. } \\
\text { ventriconvexa, M. reticulata, Ziziphocypris simakovi, } \\
\text { Rhinocypris tuberculata } \\
\text { Charophytes: Atopochara trivolvis, Nodoclavator } \\
\text { puchanghensis }\end{array}$ & Apt.-Alb. \\
\hline Bashahe Fm & $\begin{array}{ll}\text { Reddish-purple, eolian } \\
\text { sandstone and sandy } \\
\text { mudstone }\end{array}$ & no fossils & Cen.-Tur. \\
\hline Mankuanhe Fm & $\begin{array}{l}\text { Red to purple, sandy } \\
\text { mudstone, and mudstone }\end{array}$ & $\begin{array}{lccr}\text { Ostracodes: } & \text { Eucypris } & \text { anluensis, } & \text { Cypridea } \\
\text { zhengdongensis, } & \text { C. } & \text { cavernosa, } & \text { Sinocypris } \\
\end{array}$ & Con.-Maas \\
\hline
\end{tabular}




\begin{tabular}{|l|l|l|l|}
\hline & & $\begin{array}{l}\text { zhengdongensis, S. reniformis,Talicypridea subparallela, } \\
\text { T. xishuangbananensis, T. amoena } \\
\text { Charophytes: Peckichara dongyangensis, Charites } \\
\text { tenuisa }\end{array}$ & \\
\hline
\end{tabular}

\section{Khorat Basin}

\begin{tabular}{|c|c|c|c|}
\hline Formation & Lithology & Fossil assemblage & Age \\
\hline $\begin{array}{ll}\text { Phu } & \text { Kradong } \\
\text { Fm } & \\
\end{array}$ & $\begin{array}{l}\text { Reddish brown, sandstone, } \\
\text { siltstone, and mudstone }\end{array}$ & $\begin{array}{l}\text { Pollen and Spores: Cyathidites minor, Baculati- } \\
\text { sporites commaumensis, Corollina simplex }\end{array}$ & E. Cretaceous \\
\hline Phra Wihan Fm & $\begin{array}{l}\text { Whitish-grey, conglomerate, } \\
\text { sandstone, mudstone, and } \\
\text { lignites }\end{array}$ & $\begin{array}{l}\text { Pollen and Spores: Cicatricosisporites augustus, } \\
\text { Dicheiropollis etruscus, Corollina spp., Araucariacites } \\
\text { australis, Ischyosporites cf. variegatus, Gleichenidites } \\
\text { senonicus, Laevigatosporites sp., Perinopollenites } \\
\text { elatoides, Callialasporites dampieri, Anaplanisporites } \\
\text { dawsonensis, Apiculatisporites spp., Osmundacidites } \\
\text { wellmanii, Todisporites minor, Kraeuselisporites sp., } \\
\text { Concavissmisporites sp. }\end{array}$ & Ber.-Brm. \\
\hline Sao Khua Fm & $\begin{array}{l}\text { Reddish brown, sandstone, } \\
\text { siltstone, and mudstone }\end{array}$ & $\begin{array}{l}\text { Pollen and Spores: Vitreisporites cf. pallidus, } \\
\text { Cicatricosisporites spp., Cyathidites minor spp., } \\
\text { Ephedripites spp., ?Araucariacites australis }\end{array}$ & E. Cretaceous \\
\hline Phu Phan Fm & $\begin{array}{l}\text { Whitish-grey, conglomerate, } \\
\text { sandstone, mudstone }\end{array}$ & $\begin{array}{l}\text { Pollen and Spores: Corollina spp., Cyathidites } \\
\text { minor, ?Todisporites sp. }\end{array}$ & E. Cretaceous \\
\hline Khok Kruat Fm & $\begin{array}{l}\text { Reddish brown, sandstone, } \\
\text { siltstone, and mudstone }\end{array}$ & Pollen and Spores: no data presented* & Apt.? \\
\hline $\begin{array}{l}\text { Maha Sarakhan } \\
\text { Fm }\end{array}$ & $\begin{array}{l}\text { Reddish, sandstone, siltstone, } \\
\text { salts, gypsums, and } \\
\text { anhydrites }\end{array}$ & Pollen and Spores: no data presented* & Alb.?-Cen.? \\
\hline Phu Thok Fm & $\begin{array}{l}\text { Reddish, eolian sandstone and } \\
\text { siltstone }\end{array}$ & no fossils & Apt.-Tur.? \\
\hline Phu Khat Fm & $\begin{array}{l}\text { Reddish to whitish grey, } \\
\text { sandstone and mudstone }\end{array}$ & no fossils & L. Cretaceous \\
\hline
\end{tabular}

*Palynological age estimatioins of the Khok Kruat and Maha Sarakham Formations are quoted by Sattayarak et al. (1991a,b). However, no palyno-fossil assemblage data were presented. 
Table S2: Stratigraphic compilation of the climate-sensitive sediments with special emphasis on desert (eolian dune) deposits cited in Figure 3.

\begin{tabular}{|c|c|c|c|c|c|}
\hline $\begin{array}{l}\text { Locali } \\
\text { ty No. }\end{array}$ & Basin & Formation & Lithology & Age & References \\
\hline 1 & $\begin{array}{l}\text { Gobi basin, } \\
\text { Mongolia }\end{array}$ & $\begin{array}{l}\text { Khukhteeg and } \\
\text { Bayanshiree Fms }\end{array}$ & $\begin{array}{l}\text { Whitish-grey, conglomerate, } \\
\text { sandstone, mudstone, lignite, and } \\
\text { Reddish-brown to whitish-grey, } \\
\text { conglomerate, sandstone, mudstone }\end{array}$ & $\begin{array}{l}\text { Alb. } \\
\text { Cen.-San. }\end{array}$ & $\begin{array}{l}\text { Jerzykiewicz and Russell, } \\
\text { 1991; } \\
\text { Khand et al., } 2000\end{array}$ \\
\hline 2 & $\begin{array}{l}\text { Subei basin, } \\
\text { China }\end{array}$ & $\begin{array}{l}\text { Gecun and Pukou } \\
\text { Fms }\end{array}$ & $\begin{array}{l}\text { Reddish-orange to greenish-grey, } \\
\text { sandstone, mudstone, and } \\
\text { Reddish-purple to grey, pebbly } \\
\text { sandstone }\end{array}$ & $\begin{array}{l}\text { Apt.-Alb. } \\
\text { Cen.-Con. }\end{array}$ & $\begin{array}{l}\text { Jiang and Li, 1996; } \\
\text { Hao et al., } 2000\end{array}$ \\
\hline 3 & $\begin{array}{l}\text { Jianghan basin, } \\
\text { China }\end{array}$ & $\begin{array}{l}\text { Wulong and } \\
\text { Luojinghu Fms }\end{array}$ & $\begin{array}{l}\text { Reddish to yellowish-grey, } \\
\text { conglomerate, sandstone, mudstone } \\
\text { and Reddish-purple to grey, } \\
\text { conglomerate, sandstone }\end{array}$ & $\begin{array}{l}\text { Alb. } \\
\text { Cen.-Tur. }\end{array}$ & $\begin{array}{l}\text { Jiang and Li, 1996; } \\
\text { Hao et al., } 2000\end{array}$ \\
\hline 4 & $\begin{array}{l}\text { Sichuan basin, } \\
\text { China }\end{array}$ & Jiaguan Fm & $\begin{array}{l}\text { Reddish-purple, fine- } \\
\text { medium-grained, eolian sandstone }\end{array}$ & Apt.-Tur. & Jiang et al., 2001 \\
\hline 5 & $\begin{array}{l}\text { Simao basin, } \\
\text { China }\end{array}$ & $\begin{array}{l}\text { Nanxin and } \\
\text { Bashahe Fms }\end{array}$ & $\begin{array}{l}\text { Reddish-purple, sandstone, } \\
\text { mudstone and Reddish-purple, } \\
\text { eolian sandstone, sandy mudstone }\end{array}$ & $\begin{array}{l}\text { Apt.-Alb. to } \\
\text { Cen.-Tur. }\end{array}$ & Jiang et al., 2001 \\
\hline 6 & $\begin{array}{l}\text { Khorat basin, } \\
\text { Thailand }\end{array}$ & $\begin{array}{l}\text { Maha Sarakhan } \\
\text { and Phu Thok Fms }\end{array}$ & $\begin{array}{l}\text { Reddish, sandstone, siltstone, salts, } \\
\text { gypsums, and Reddish, eolian } \\
\underline{\text { sandstone, siltstone }}\end{array}$ & Apt.-Tur.? & $\begin{array}{l}\text { Imsamut, 1996; } \\
\text { Hasegawa et al., } 2010\end{array}$ \\
\hline 7 & $\begin{array}{l}\text { Iberian basin, } \\
\text { Spain }\end{array}$ & $\begin{array}{l}\text { Escucha and } \\
\text { Utrillas Fms }\end{array}$ & $\begin{array}{l}\text { Fine-grained eolian sandstone, } \\
\text { siltstone, mudstone }\end{array}$ & Alb.-Cen. & $\begin{array}{l}\text { Rodriguez-Lopez et al., } \\
2006,2008\end{array}$ \\
\hline 8 & $\begin{array}{l}\text { British } \\
\text { Columbia, } \\
\text { Canada }\end{array}$ & Boulder Creek Fm & $\begin{array}{l}\text { Grayish very fine-grained } \\
\text { sandstone, Greenish-gray mudstone, } \\
\text { sphaerosiderite- bearing paleosol }\end{array}$ & Alb.-Cen. & $\begin{array}{l}\text { Leckie et al., 1989; } \\
\text { Ufnar et al., } 2005\end{array}$ \\
\hline 9 & $\begin{array}{l}\text { North Alberta, } \\
\text { Canada }\end{array}$ & Peace River Fm & $\begin{array}{l}\text { Grayish sandstone, mudstone, } \\
\text { sphaerosiderite-bearing paleosol }\end{array}$ & Alb.-Cen. & Ufnar et al., 2005 \\
\hline 10 & $\begin{array}{l}\text { South Alberta, } \\
\text { Canada }\end{array}$ & $\begin{array}{l}\text { Mill Creek and } \\
\text { Bow Island Fms }\end{array}$ & $\begin{array}{l}\text { Reddish and grayish-greenish } \\
\text { sandstone, pale yellow to dark red } \\
\text { mudstone, paleosol }\end{array}$ & Alb. & $\begin{array}{l}\text { McCarthy et al., 1997, } \\
1999\end{array}$ \\
\hline 11 & $\begin{array}{l}\text { Ontario basin, } \\
\text { Canada }\end{array}$ & Mattagami Fm & $\begin{array}{l}\text { Varicolored conglomerate, } \\
\text { sandstone, mudstone, lignite }\end{array}$ & $\begin{array}{l}\text { Apt.-Alb. to } \\
\text { Cen. }\end{array}$ & White et al., 2000 \\
\hline 12 & $\begin{array}{l}\text { Western Iowa } \\
\text { basin }\end{array}$ & $\begin{array}{l}\text { Dakota and Swan } \\
\text { River Fms }\end{array}$ & $\begin{array}{l}\text { Grayish sandstone, mudstone, } \\
\text { sphaerosiderite-bearing paleosol }\end{array}$ & Alb.-Cen. & $\begin{array}{l}\text { Ludvigson et al., 1998; } \\
\text { White et al., } 2000\end{array}$ \\
\hline 13 & $\begin{array}{l}\text { Southwest } \\
\text { Utah basin }\end{array}$ & $\begin{array}{l}\text { Upper part of the } \\
\text { Dakota Fm }\end{array}$ & $\begin{array}{l}\text { Varicolored conglomerate, } \\
\text { sandstone, mudstone, coal }\end{array}$ & Cen.-Tur. & $\begin{array}{l}\text { Laurin and Sageman, } \\
\text { 2007; } \\
\text { Barclay et al., } 2010\end{array}$ \\
\hline 14 & $\begin{array}{l}\text { New Mexico } \\
\text { basin }\end{array}$ & $\begin{array}{l}\text { Sarten and Moreno } \\
\text { Hill Fms }\end{array}$ & $\begin{array}{l}\text { Reddish to pale yellowish } \\
\text { sandstone, mudstone, paleosol }\end{array}$ & $\begin{array}{l}\text { Alb.-Cen. to } \\
\text { Tur. }\end{array}$ & Mack, 1992 \\
\hline 15 & $\begin{array}{l}\text { Araripe basin, } \\
\text { Brazil }\end{array}$ & Santana Fm & Sandstone, mudstone, black shale & Alb. & Heimhofer et al., 2008 \\
\hline 16 & $\begin{array}{l}\text { Salta basin, } \\
\text { Algentina }\end{array}$ & La Yesera Fm & $\begin{array}{l}\text { Reddish-brown conglomerate, } \\
\text { sandstone, siltstone, mudstone }\end{array}$ & Apt.-Alb. & Marquillas et al., 2005 \\
\hline 17 & $\begin{array}{l}\text { Neuquen basin, } \\
\text { Algentina }\end{array}$ & Lohan Cura Fm & $\begin{array}{l}\text { Reddish-brown to greenish gray } \\
\text { conglomerate, sandstone, siltstone, }\end{array}$ & Apt.-Alb. & Leanza et al., 2004 \\
\hline
\end{tabular}




\begin{tabular}{|c|c|c|c|c|c|}
\hline & & & shale & & \\
\hline 18 & $\begin{array}{l}\text { Orange basin, } \\
\text { Namibia }\end{array}$ & $\begin{array}{l}\text { Alb. to Cen. } \\
\text { succession }\end{array}$ & $\begin{array}{l}\text { Whitish conglomerate, sandstone, } \\
\text { siltstone, mudstone }\end{array}$ & Alb.-Cen. & $\begin{array}{l}\text { Stevenson and McMillan, } \\
\text { 2004; }\end{array}$ \\
\hline 19 & $\begin{array}{l}\text { Tendaguru } \\
\text { basin, Tanzania }\end{array}$ & Makonde Fm & $\begin{array}{l}\text { Reddish to purple, conglomerate, } \\
\text { fine- to medium-grained sandstone, } \\
\text { siltstone, mudstone }\end{array}$ & Apt.-Alb. & Bussert et al., 2009 \\
\hline 20 & $\begin{array}{l}\text { Saurashtra } \\
\text { basin, India }\end{array}$ & $\begin{array}{l}\text { Than and } \\
\text { Wadhwan Fms }\end{array}$ & $\begin{array}{l}\text { Grayish sandstone, mudstone, coal } \\
\text { and Reddish conglomerate, } \\
\text { sandstone, mudstone, limestone }\end{array}$ & E. Cretaceous & Aslam, 1992 \\
\hline 21 & $\begin{array}{l}\text { Gippsland } \\
\text { basin, Australia }\end{array}$ & Wonthaggi Fm & $\begin{array}{l}\text { Volcanogenic sandstone, mudstone, } \\
\text { coal }\end{array}$ & Apt.-Alb. & $\begin{array}{l}\text { Douglas and Williams, } \\
\text { 1982; } \\
\text { Tosolini et al., } 2002\end{array}$ \\
\hline 22 & $\begin{array}{l}\text { Gobi basin, } \\
\text { Mongolia }\end{array}$ & $\begin{array}{l}\text { Djadokhta, } \\
\text { Barungoyot, } \\
\text { Dzunmod Fms }\end{array}$ & $\begin{array}{l}\text { Reddish brown to whitish grey, } \\
\text { eolian sandstone, sandy mudstone, } \\
\text { calcrete }\end{array}$ & Camp.-Maas. & $\begin{array}{l}\text { Jerzykiewicz and Russell, } \\
\text { 1991; } \\
\begin{array}{l}\text { Hasegawa et al., in } \\
\text { submitted }\end{array}\end{array}$ \\
\hline 23 & $\begin{array}{l}\text { Ordos basin, } \\
\text { China }\end{array}$ & Tagaimiao Fm & $\begin{array}{l}\text { Red to orange, fine- to } \\
\text { medium-grained, eolian sandstone }\end{array}$ & San.-Camp. & $\begin{array}{l}\text { Jiang and Li, 1996; } \\
\text { Hao et al., } 2000\end{array}$ \\
\hline 24 & $\begin{array}{l}\text { Tarim basin, } \\
\text { China }\end{array}$ & Subashi Fm & $\begin{array}{l}\text { Whitish-grey to reddish, medium to } \\
\text { coarse-grained sandstone, mudstone }\end{array}$ & Camp.-Maas. & $\begin{array}{l}\text { Jiang and Li, 1996; } \\
\text { Hao et al., } 2000\end{array}$ \\
\hline 25 & $\begin{array}{l}\text { Subei basin, } \\
\text { China }\end{array}$ & Chishan Fm & $\begin{array}{l}\text { Reddish-purple to greenish-grey, } \\
\text { sandstone, mudstone }\end{array}$ & San.-Maas. & $\begin{array}{l}\text { Jiang and Li, 1996; } \\
\text { Hao et al., } 2000\end{array}$ \\
\hline 26 & $\begin{array}{l}\text { Jianghan basin, } \\
\text { China }\end{array}$ & Paomagang Fm & $\begin{array}{l}\text { Reddish to whitish grey, sandstone, } \\
\text { mudstone }\end{array}$ & Camp.-Maas. & $\begin{array}{l}\text { Jiang and Li, 1996; } \\
\text { Hao et al., } 2000\end{array}$ \\
\hline 27 & $\begin{array}{l}\text { Sichuan basin, } \\
\text { China }\end{array}$ & Guankou Fm & $\begin{array}{l}\text { Red to purple, fine-grained } \\
\text { sandstone, mudstone, gypsum }\end{array}$ & Con.-Maas. & Jiang et al., 2001 \\
\hline 28 & $\begin{array}{l}\text { Simao basin, } \\
\text { China }\end{array}$ & Mankuanhe Fm & $\begin{array}{l}\text { Red to purple, sandy mudstone, } \\
\text { mudstone }\end{array}$ & Con.-Maas. & Jiang et al., 2001 \\
\hline 29 & $\begin{array}{l}\text { Khorat basin, } \\
\text { Thailand }\end{array}$ & Phu Khat Fm & $\begin{array}{l}\text { Reddish to whitish grey, sandstone, } \\
\text { mudstone }\end{array}$ & L. Cretaceous & Hasegawa et al., 2010 \\
\hline 30 & $\begin{array}{l}\text { South Alberta, } \\
\text { Canada }\end{array}$ & $\begin{array}{l}\text { Belly River and } \\
\text { Willow Creek Fms }\end{array}$ & $\begin{array}{l}\text { Reddish brown to whitish grey, } \\
\text { conglomerate, sandstone, mudstone, } \\
\text { calcrete }\end{array}$ & Camp.-Maas. & $\begin{array}{l}\text { Jerzykiewicz and Sweet, } \\
\text { 1988; }\end{array}$ \\
\hline 31 & $\begin{array}{l}\text { Western } \\
\text { Montana basin, } \\
\text { Canada }\end{array}$ & Two Medicine Fm & $\begin{array}{l}\text { Reddish brown to whitish grey, } \\
\text { conglomerate, sandstone, mudstone, } \\
\text { calcrete }\end{array}$ & Camp.-Maas. & Lorenz, 1981 \\
\hline 32 & $\begin{array}{l}\text { Eastern } \\
\text { Montana basin, } \\
\text { Canada }\end{array}$ & Hell Creek Fm & $\begin{array}{l}\text { Varicolored conglomerate, } \\
\text { sandstone, mudstone, coal }\end{array}$ & Maas. & $\begin{array}{l}\text { Retallack, 1994; } \\
\text { Johnson et al., } 2002\end{array}$ \\
\hline 33 & $\begin{array}{l}\text { North Dakota } \\
\text { basin }\end{array}$ & Hell Creek Fm & $\begin{array}{l}\text { Varicolored conglomerate, } \\
\text { sandstone, mudstone, coal }\end{array}$ & Maas. & $\begin{array}{l}\text { Fastovsky } \\
\text { McSweeney, } 1987 \\
\text { Johnson et al., } 2002\end{array}$ \\
\hline 34 & $\begin{array}{l}\text { Southwest } \\
\text { Utah basin }\end{array}$ & $\begin{array}{l}\text { Wahweap and } \\
\text { Kaiparowits Fms }\end{array}$ & $\begin{array}{l}\text { Varicolored conglomerate, } \\
\text { sandstone, mudstone }\end{array}$ & Camp.-Maas. & Lawton et al., 2003 \\
\hline 35 & $\begin{array}{l}\text { New Mexico } \\
\text { basin }\end{array}$ & MacRae Fm & $\begin{array}{l}\text { Reddish-brown to greyish, } \\
\text { conglomerate, sandstone, mudstone, } \\
\text { calcrete }\end{array}$ & Maas. & Buck and Mack, 1995 \\
\hline 36 & $\begin{array}{l}\text { Western Texas } \\
\text { basin }\end{array}$ & $\begin{array}{l}\text { Aguja and Javelina } \\
\text { Fms }\end{array}$ & $\begin{array}{l}\text { Reddish purple to grayish, } \\
\text { sandstone, mudstone, paleosol }\end{array}$ & Maas. & Lehman, 1989, 1990 \\
\hline 37 & Salta basin, & Lecho Fm & Whitish, fine- to medium-grained, & Maas. & Marquillas et al., 2005 \\
\hline
\end{tabular}




\begin{tabular}{|c|c|c|c|c|c|}
\hline & Algentina & & eolian sandstone & & \\
\hline 38 & $\begin{array}{l}\text { Bauru basin, } \\
\text { Brazil }\end{array}$ & $\begin{array}{l}\text { Rio Parana Fm, } \\
\text { Caiua Gp. }\end{array}$ & $\begin{array}{l}\text { Fine- to medium-grained, quartz } \\
\text { eolian sandstone }\end{array}$ & Con.-Maas. & Fernandes et al., 2007 \\
\hline 39 & $\begin{array}{l}\text { Parana basin, } \\
\text { Brazil }\end{array}$ & Marilia Fm & $\begin{array}{l}\text { Medium- to coarse-grained, } \\
\text { quartz-feldspar eolian sandstone, } \\
\text { calcretes }\end{array}$ & Maas. & $\begin{array}{l}\text { Goldberg and Garcia, } \\
2000\end{array}$ \\
\hline 40 & $\begin{array}{l}\text { Neuquen basin, } \\
\text { Algentina }\end{array}$ & $\begin{array}{l}\text { Anacleto and Allen } \\
\text { Fms }\end{array}$ & $\begin{array}{l}\text { Reddish to greenish-gray, } \\
\text { conglomerate, sandstone, siltstone, } \\
\text { mudstone }\end{array}$ & San.-Maas. & Leanza et al., 2004 \\
\hline 41 & $\begin{array}{l}\text { Anambra } \\
\text { basin, Namibia }\end{array}$ & $\begin{array}{l}\text { Mamu and Ajali } \\
\text { Fms }\end{array}$ & $\begin{array}{l}\text { Conglomerate, sandstone, shale, } \\
\text { coal }\end{array}$ & Camp.-Maas. & Tuttle, 1999 \\
\hline 42 & $\begin{array}{l}\text { Congo basin, } \\
\text { Angola }\end{array}$ & Nsele Gp & $\begin{array}{lcr}\begin{array}{l}\text { Medium- } \\
\text { sandstone, }\end{array} & \text { to } & \text { colian } \\
\text { texture } & & \end{array}$ & L.Cretaceous & Giresse, 2005 \\
\hline 43 & $\begin{array}{l}\text { Orange basin, } \\
\text { Namibia }\end{array}$ & $\begin{array}{l}\text { Upper Santonian } \\
\text { succession }\end{array}$ & Brownish sandstone, mudstone & L. Santonian & $\begin{array}{l}\text { Stevenson and McMillan, } \\
\text { 2004; }\end{array}$ \\
\hline 44 & $\begin{array}{l}\text { Dongargaonba } \\
\text { sin, India }\end{array}$ & Lameta Fm & $\begin{array}{l}\text { Reddish to greenish, conglomerate, } \\
\text { sandstone, mudstone, calcrete }\end{array}$ & Maas. & $\begin{array}{l}\text { Mohabey et al., 1993; } \\
\text { Mohabey, } 1996\end{array}$ \\
\hline 45 & $\begin{array}{l}\text { Gippsland } \\
\text { basin, Australia }\end{array}$ & Latrobe Gp. & $\begin{array}{l}\text { Conglomerate, sandstone, } \\
\text { mudstone, coal, volcanics }\end{array}$ & Camp.-Maas. & $\begin{array}{l}\text { Wagstaff et el., 2006; } \\
\text { Gallagher et al., } 2008\end{array}$ \\
\hline
\end{tabular}




\section{Supplementary Methods:}

\section{Paleoposition of eolian sandstone deposits in Asian interior basins}

Paleolatitude and rotation estimates of the studied basins, which are reconstructed based on paleomagnetic data (Cheng et al., 1988; Zhuang, 1988; Otofuji et al., 1990; Enkin et al., 1991; Zheng et al., 1991; Chen et al., 1993; Huang and Opdyke, 1993; Hankard et al., 2005; Charusiri et al., 2006; Fig. 1 and Table 1), are the critical basis for the present study which demonstrate that the location of the subtropical high-pressure belt changed significantly during the Cretaceous. Based on the obtained paleomagnetic data sets, paleolatitudes of the North China block (Ordos Basin: N32.6 ${ }^{\circ}-41.0^{\circ}$; Cheng et al., 1988; Zheng et al., 1991) and the South China block (Sichuan Basin: N25.5 $-29.6^{\circ}$; Zhuang et al., 1988; Enkin et al., 1991) were different by more than $5^{\circ}$ during the Cretaceous (Fig. 1 and Table 1). The Gobi Basin of southern Mongolia was located much higher latitude during the Cretaceous (N44. $0^{\circ}-46.1^{\circ}$; Hankard et al., 2005). Reconstruction of the paleoposition of Indochina block during the Cretaceous, prior to the India-Asia collision, has been controversial. For example, Chen et al. (1992) argued that both Indochina block and South China block were located between $\mathrm{N}^{\circ} 0^{\circ}$ and $\mathrm{N} 30^{\circ}$ during the Cretaceous, based on the paleomagnetic data reported by Yang and Basse (1993). However, the paleomagnetic data reported by Yang and Basse (1993) is for the upper Triassic to the lower Cretaceous deposits in the Khorat Basin (Indochina block) in northeastern Thailand, and no paleomagnetic data of the mid- to upper Cretaceous deposits, which contain eolian sandstone deposits in this area, was reported. On the other hand, Charusiri et al. (2006) conducted the paleomagnetic study on the mid- to upper Cretaceous deposits in the Khorat Basin, northeastern Thailand for the first time. They concluded that the Khorat Basin was located between N16.3 ${ }^{\circ}$ and $21.6^{\circ}$ during the mid- to late Cretaceous time (Charusiri et al., 2006), which was much lower than that of South China block (N25.5-29.6 ${ }^{\circ}$; Zhuang et al., 1988; Enkin et al., 1991). Therefore, eolian sandstones were distributed in ca. $\mathrm{N} 30^{\circ}-40^{\circ}$ (Ordos and Tarim basins) during the early Cretaceous, shifted southwards to ca. $\mathrm{N} 20^{\circ}-30^{\circ}$ (Sichuan and Khorat basins) during the mid-Cretaceous, and shifted northwards again to ca. $\mathrm{N} 30^{\circ}-45^{\circ}$ (Gobi and Ordos basins) during the late Cretaceous, implying that the significant latitudinal shifts of the distribution of eolian sandstone deposits have occurred between the early, mid-, and late Cretaceous (Fig. 1). 


\section{Age constraints for eolian sandstone formations in Asian interior basins}

Although non-marine strata generally have chronostratigraphic uncertainties, our selected data sets of eolian sandstone formations in the Asian interior basins have good age controls based on magnetostratigraphy and/or biostratigraphy (Li, 1982; Jerzykiewicz and Russell, 1991; Jiang and Li, 1996; Racey et al., 1996; Hao et al., 2000; Khand et al., 2000; Meesok, 2000; Jiang et al., 2001; Chen et al., 2006; Sha, 2007; Fig. 1 and Table S1), including the results of our magnetostratigraphic studies (Imsamut, 1996; Pan et al., 2004; Hasegawa et al., 2010; Figs. S1, S2). Paleontological age constraints provide a starting point for the ages of the eolian sandstone formations cited in this study. A relatively rich record of fossil ostracods, charophytes, plants, pollens, and spores has been collected from the studied sites (Table S1). Particularly important are the ages of the eolian sandstone formations in Sichuan Basin and Khorat Basin (the Jiaguan Formation and the Phu Thok Formation), because they provide the critical time constraints on the low latitude desert distribution during the mid-Cretaceous. The ages of these formations are well constrained by our magnetostratigraphic data (Imsamut, 1996; Pan et al., 2004; Hasegawa et al., 2010) in conjunction with paleontological age constraints as described below.

The age of the Jiaguan Formation in Sichuan Basin is assigned to the Aptian-Turonian based on the ostracod's biostratigraphy of the Jiaguan Formation, which yields age-diagnostic ostracodes such as Ziziphocypris orbita, Cypridea (Bisulcocypridea) sp. (Cenomanian-Turonian), and Latonia (Monosulcocypris) spp. (Aptian) (Li, 1982; Hao et al., 2000; Chen et al., 2006; Table S1). Such age constraint is consistent with the ostracod's biostratigraphy of the underlying Tianmashan Formation (Berriasian-Barremian) and overlying Guankou Formation (Coniacian-Maastrichtian). In addition, the obtained paleomagnetic polarity sequence of the Jiaguan Formation reveals short repetition of reverse-normal-reverse polarity changes in its lowermost part and thick normal polarity zone in its main part (Pan et al., 2004), which correlate best with chron M1r to superchron C34n of the geomagnetic polarity time scale (GPTS; Gradstein et al., 2004). These results suggest that deposition of the Jiaguan Formation began approximately at $127 \mathrm{Ma}$ and ended no later than ca. 84 Ma (from late Barremian-early Aptian to no later than late Santonian) (Pan et al., 2004; Fig. S1).

Age constraints of the Phu Thok Formation in Khorat Basin are provided by the palynological evidences of the underlying strata, the lignite-bearing Phra Wihan Formation, which yields 
age-diagnostic palyno-fossils such as Cicatricosisporites augustus indicating the age no older than Berriasian and Dicheiropollis etruscus indicating the age of Barremian (Racey et al., 1996). Thus, age of the Phra Wihan Formation is assigned to the early Cretaceous (Berriasian-Barremian), and the age of the overlying Phu Thok Formation is younger than Barremian age (Racey et al., 1996; Meesok, 2000; DMR, 2001; Charusiri et al., 2006; Table S1). Our newly established paleomagnetic polarity sequence of the Phu Thok Formation reveals short repetition of reverse-normal-reverse polarity changes in its lowermost part and thick normal polarity zone in its main part (Imsamut, 1996; Hasegawa et al., 2010; Fig. S2), which can be correlated with chron M1n to superchron C34n of the GPTS (Gradstein et al., 2004). Consequently, deposition of the Phu Thok Formation is interpreted as having started approximately at $126 \mathrm{Ma}$ and ended no younger than ca. $84 \mathrm{Ma}$ (from late Barremian-early Aptian to no younger then late Santonian), which is approximately the same age as the Jiaguan Formation in Sichuan Basin. In summary, the low latitude deserts in Asia emerged between late Barremian and early Aptian and disappeared between Turonian and Coniacian according to the magnetostratigraphic correlations and paleontological age constraints of the eolian sandstone formations in the Sichuan Basin (Jiaguan Formation) and the Khorat Basin (Phu Thok Formation) given above (Fig. 1). 


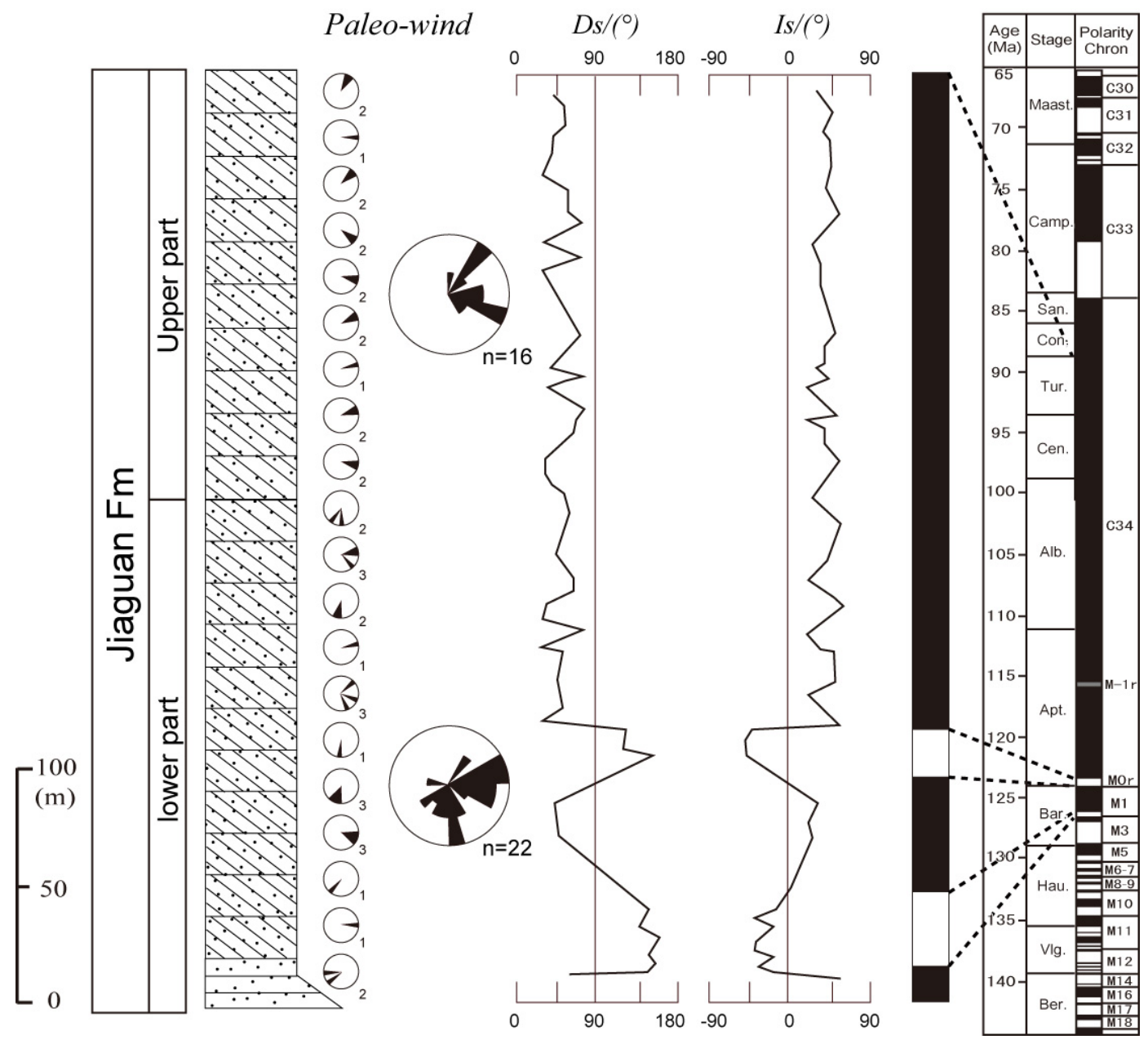

Fig. S1: Lithostratigraphic column, paleowind direction data, and magnetic polarity sequence of the eolian sandstone deposits (Jiaguan Formation) in Sichuan Basin, south China (Jiang et al., 1999; Pan et al., 2004; Hasegawa et al., 2010), and their correlation to the geomagnetic polarity time scale (GPTS) of the geological time scale 2004 (Gradstein et al., 2004). Magnetic polarity zones are shown by black and white bars for normal and reversed polarities. 


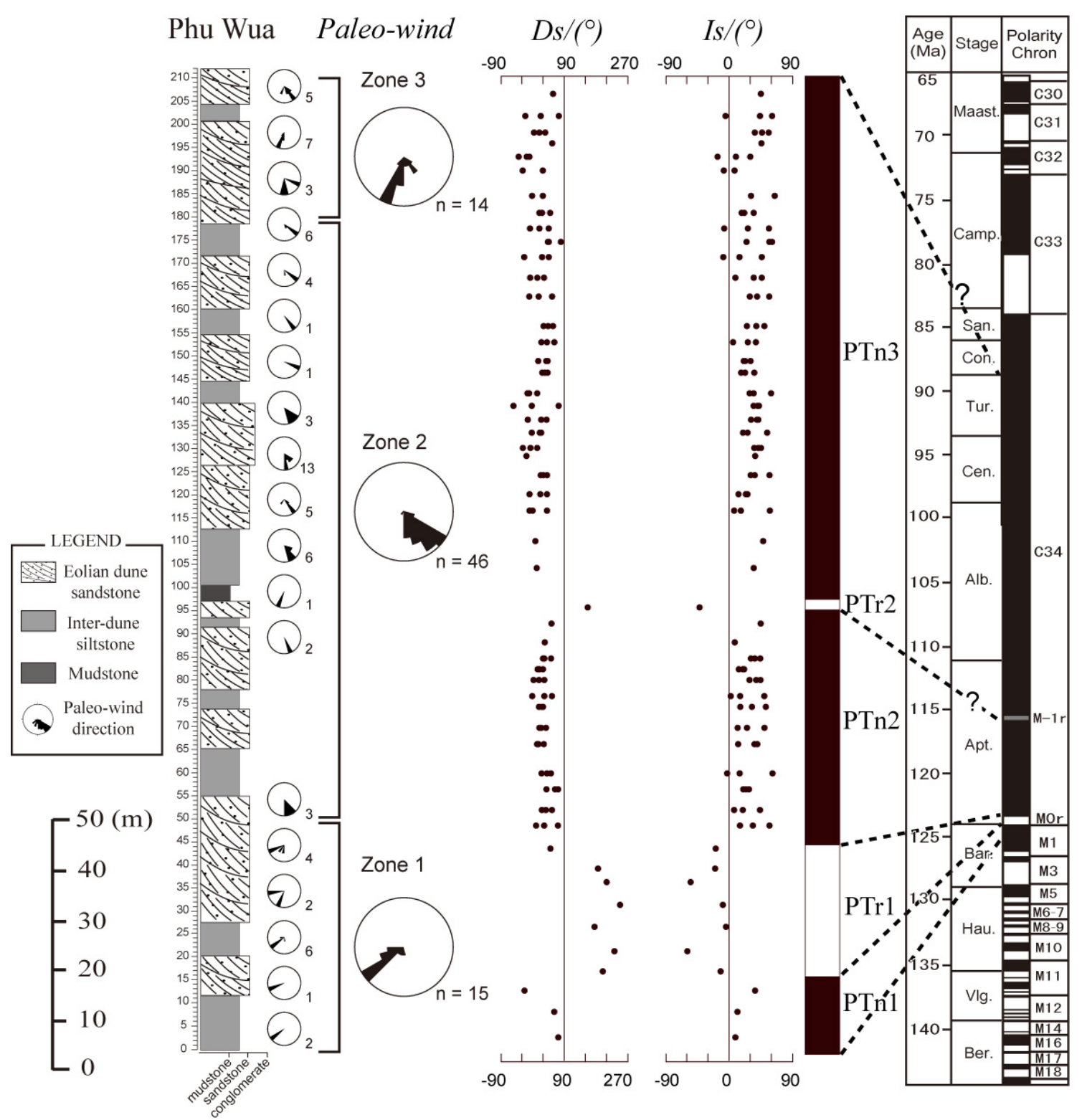

Fig. S2: Lithostratigraphic column, paleowind direction data, and magnetic polarity sequence of the eolian sandstone deposits (Phu Thok Formation) in the Khorat Basin, northeast Thailand (Imsamut, 1996; Hasegawa et al., 2010), and their correlation to the GPTS (Gradstein et al., 2004). Magnetic polarity zones are shown by black (white) bars for normal (reversed) polarity. 


\section{Supplementary References}

Aslam, M., 1992. Delta plain coal deposits from the Than Formation of the Early Cretaceous Saurashtra basin, Gujarat, western India. Sedimentary Geology 81, 181-193.

Barclay, R.S., McElwain, J.C., Sageman, B.B., 2010. Carbon sequestration activated by a volcanic CO2 pulse during Ocean Anoxic Event 2. Nature Geoscience 3, 205-208.

Buck, B.J., Mack, G.H. 1995. Latest Cretaceous (Maastrichtian) aridity indicated by paleosols in the McRae Formation, south-central New Mexico. Cretaceous Research 16, 559-572.

Bussert, R., Heinrich, W.D., Aberhan, M., 2009. The Tendaguru Formation (Late Jurassic to Early Cretaceous, southern Tanzania): definition, palaeoenvironments, and sequence stratigraphy. Fossil Record 12, 141-174.

Douglas, J.G., Williams, G.E., 1982. Southern polar forests: The Early Cretaceous floras of Victoria and their palaeoclimatic significance. Palaeogeography, Palaeoclimatology, Palaeoecology 39, 171-185.

Fastovsky, D.E., McSweeney, K., 1987. Paleosols spanning the Cretaceous-Paleogene transition, eastern Montana and western North Dakota. Geological Society of America Bulletin 99, 66-77.

Gallagher, S.J., Wagstaff, B.E., Baird, J.G., Wallace, M.W., Li, C.L., 2008. Southern high latitude climate variability in the Late Cretaceous greenhouse world. Global and Planetary Change 60, 351-364.

Hasegawa, H., Suganuma, Y., Seike, K., Tada, R., Ichinnorov, N., Badamgarav, D., Khand, Y., in submitted. Magnetostratigraphy and depositional environments of Upper Cretaceous deposits in the Gobi Basin, southern Mongolia: implications for desert development in mid-latitude Asia. (submitted to Journal of Asian Earth Sciences)

Heimhofer, U., Hesselbo, S.P., Pancost, R.D., Martill, D.M., Hochuli, P.A., Guzzo, J.V.P., 2008. Evidence for photic-zone euxinia in the Early Albian Santana Formation (Araripe Basin, NE Brazil). Terra Nova 20, 347-354.

Jerzykiewicz, T., Sweet, A.R., 1988. Sedimentological and palynological evidence of regional climatic changes in the Campanian to Paleocene sediments of the Rocky Mountain Foothills, Canada. Sedimentary Geology 59, 29-76.

Jiang, X.S., Pan, Z.X., Fu, Q.P., 1999. The variations of palaeowind direction of the Cretaceous desert in the Sichuan Basin and their significance. Sedimentary Facies and Palaeogeography 19, 1-11.

Johnson, K.R., Nichols, D.J., Hartman, J.H., 2002. Hell Crek Formation: A 2001 synthesis. 
Geological Society of America Special Paper 361, 503-510.

Laurin, J., Sageman, B., 2007. Cenomanian_Turonian coastal record in SW Utah, USA: Orbital scale transgressive-regressive events during Oceanic Anoxic Event II. Journal of Sedimentary Research 77, 731-756.

Lawton, T.F., Polloch, S.L., Robinson, R.A.J., 2003. Integrating sandstone petrology and nonmarine sequence stratigraphy: Application to the late cretaceous fluvial systems of southwestern Utah, USA. Journal of Sedimentary Research 73, 389-406.

Leanza, H.A., Apesteguia, A., Novas, F.E., de la Fuente, M.S., 2004. Cretaceous terrestrial beds from the Neuquén Basin (Argentina) and their tetrapod assemblages. Cretaceous Research 25, 61-87.

Leckie, D., Fox, C., Tarnocai, C., 1989. Multiple paleosols of the late Albian Boulder Creek Formation, British Columbia, Canada: Sedimentology 36, 307-323.

Lehman, T.M., 1989. Upper Cretaceous (Maastrichtian) Paleosols in Trans-Pecos Texas. Geological Society of America Bulletin 101, 188-203.

Lehman, T.M., 1990. Paleosols and the Cretaceous/Tertiary transition in the Big Bend region of Texas. Geology 18, 362-364.

Lorenz, J.C., 1981. Sedimentary and tectonic history of the Two Medicine Formation, Late Cretaceous (Campanian), northwestern Montana. Ph.D. Thesis, Princeton University, Princeton, N.J., pp.214 .

Mack, G.H., 1992. Paleosols as an indicator of climatic-change at the early-late Cretaceous boundary, Southwestern New Mexico. Journal of Sedimentary Petrology 62, 483-494.

McCarthy, P.J., Martini, I.P., Leckie, D.A., 1997. Anatomy and evolution of a Lower Cretaceous alluvial plain: Sedimentology and palaeosols in the upper Blairmore Group, south-western Alberta, Canada. Sedimentology 44, 197-220.

McCarthy, P.J., Martini, I.P., Leckie, D.A., 1999. Pedogenic and diagenetic influences on void coating formation in Lower Cretaceous paleosols of the Mill Creek Formation, southwestern Alberta, Canada. Geoderma 87, 209-237.

Mohabey, D.M., Udhoji, S.G., Verma, K.K., 1993. Palaeontological and sedimentological observations on non-marine Lameta Formation (Upper Cretaceous) of Maharashtra, India: their palaeeoecological and palaeoenvironmental significance. Palaeogeography, Palaeoclimatology, Palaeoecology 105, 83-94.

Mohabey, D.M., 1996. A new oospecies, Megaloolithus matleyi, from the Lameta Formation (Upper Cretaceous) of Chandrapur district, Maharashtra, India, and general remarks on the 
palaeoenvironment and nesting behaviour of dinosaurs. Cretaceous Research 17, 183-196.

Racey, A., Love, M. ., Canham, A. C., Goodall, J. G. S., Polachan, S., and Jones, P. D.: Stratigraphy and reservoir potential of the Mesozoic Khorat Group, NE Thailand, J. Petrol. Geol., 19, 5-40, 1996.

Retallack, G.J., 1994. A pedotype approach to latest Cretaceous and earliest Tertiary paleosols in eastern Montana. Geological Society of America Bulletin 106, 1377-1397.

Rodriguez-Lopez, J.P., de Boer, P.L., Melendez, N., Soria, A.R., Pardo, G., 2006. Windblown desert sands in coeval shallow marine deposits: a key for the recognition of coastal ergs in the mid-Cretaceous Iberian Basin, Spain. Terra Nova 18, 314-320.

Sattayarak, N., Srigulwong, S., Patarametha, M., 1991a. Subsurface stratigraphy of the non-marine Mesozoic Khorat Group, Northern Thailand. GEOSEA VII, Bangkok, 36A.

Sattayarak, N., Polachan, S., Charusirisawad, R., 1991b. Cretaceous rock salt in the northeastern part of Thailand. GEOSEA VII, Bangkok, 36A.

Stevenson, I.R., McMillan, I.K., 2004. Incised valley fill stratigraphy of the Upper Cretaceous succession, proximal Orange Basin, Atlantic margin of southern Africa. Journal of the Geological Society, London 161, 185-208.

Tosolini, A.M.P., McLoughlin, S., Drinnan, A.N., 2002. Early Cretaceous megaspore assemblages from southeastern Australia. Cretaceous Research 23, 807-844.

Tuttle, M.L.W., Charpentier, R.R., Brownfield, M.E., 1999. The Niger Delta Petroleum System: Niger Delta Province, Nigeria, Cameroon, and Equatorial Guinea, Africa. Open-File Report, U.S. Department of the Interior, U.S. Geological Survey, pp.64.

Ufnar, D. F., Gonzalez, L. A., Ludvigson, G. A., Brenner, R. L., Witzke, B. J. Leckie, D., 2005. Reconstructing a mid-Cretaceous landscape from paleosols in western Canada. Journal of Sedimentary Research75, 984-996.

Wagstaff, B.E., Gallagher S.J.,Lanigan, K.P., 2006. Late Cretaceous palynological correlation and environmental analysis of fluvial reservoir facies of the Tuna Field, Gippsland Basin, southeast Australia. Review of Palaeobotany and Palynology 138, 165-186.

White, T.S., Witzke, B., Ludvigson, G., 2000. Evidence for an Albian Hudson Arm of the North American Cretaceous Western Interior Seaway. Geological Society of America Bulletin 112, 1342-1355.

Yang, Z., and Basse, J.: Paleomagnetic study of Permian and Mesozoic sedimentary rocks from Northern Thailand supports the exteuction model for Indochina, Earth Planet. Sc. Lett., 117, 525-552, 1993. 